\title{
De l'émotion musicale à la farce
}

Varier le rythme de la veillée pour maîtriser l'humeur (Yémen)

From Musical Emotion to Jokes. Varying the Rhythm of the Evening Gathering in order to master the Mood (Yemen)

\section{Jean Lambert}

\section{OpenEdition \\ Journals}

\section{Édition électronique}

URL : https://journals.openedition.org/clo/2076

DOI : $10.4000 /$ clo. 2076

ISSN : 2266-1816

Éditeur

INALCO

\section{Édition imprimée}

Date de publication : 1 janvier 2013

ISBN : 978-2-85831-217-7

ISSN : 0396-891X

Référence électronique

Jean Lambert, « De l'émotion musicale à la farce », Cahiers de littérature orale [En ligne], 73-74 | 2013, mis en ligne le 12 mai 2015, consulté le 01 juillet 2021. URL : http://journals.openedition.org/clo/2076 ; DOI : https://doi.org/10.4000/clo.2076

Ce document a été généré automatiquement le 1 juillet 2021.

\section{c) (1) (9)}

Cahiers de littérature orale est mis à disposition selon les termes de la Licence Creative Commons Attribution - Pas d'Utilisation Commerciale 4.0 International. 


\title{
De l'émotion musicale à la farce
}

\author{
Varier le rythme de la veillée pour maîtriser l'humeur (Yémen) \\ From Musical Emotion to Jokes. Varying the Rhythm of the Evening Gathering in \\ order to master the Mood (Yemen)
}

Jean Lambert

1 À Sanaa, au Yémen, les séances sociales, ou magyal, réunissent les hommes tous les après-midis dans des grands salons orientés vers le couchant, et elles s'accompagnent de la consommation de qat, une plante stimulante locale. La recherche d'un équilibre des humeurs et des passions (homéostasie) est un objectif majeur des participants. Elle s'appuie entre autres sur la pratique d'une rythmique du temps social basée sur des changements de rythme dans les interactions cérémonielles, mais aussi musicales. Le modèle dominant est une succession de trois phases psychologiques (théoriquement liées à la consommation du qat): une phase d'accueil émaillée de plaisanteries, une phase de discussions sérieuses et une phase de contemplation esthétique. Le passage entre ces différentes atmosphères se fait d'une manière graduelle, par une sorte de « tuilage » produisant une perception ambiguë entre la phase précédente et la suivante, jusqu'à la découverte soudaine que l'on a changé de phase. Un tel tuilage concerne aussi la perception du passage du jour à la nuit. Dans la suite musicale également, le musicien cultive une forme de tuilage entre deux mouvements se succédant, et produit une perception brouillée, comme s'il fallait éviter de faire sentir le passage et que la conscience n'en émerge qu'aposteriori. Il s'agit en somme de suspendre momentanément la perception de la durée, puis de la rétablir brusquement, ce qui contribue à accorder au temps une valeur subjective. J'avais étudié ces processus cérémoniels et musicaux en les qualifiant de "parcours obligés", marqués par la continuité, la progression et l'irréversibilité (Lambert, 1997, 46-47 ; 2004, 152).

2 Ces séances d'après-midi sont parfois suivies de séances nocturnes ou veillées, samra, principalement à l'occasion des fêtes de mariage (mais aussi des fêtes religieuses). Les samra ressemblent aux magyal par certains aspects, mais elles en diffèrent aussi, en ce qu'elles sont moins formelles et plus décontractées. De plus, l'humour et la plaisanterie 
y jouent un rôle plus important, en ponctuant la séance et en introduisant des changements de rythme dans le cérémoniel, souvent en relation avec la musique.

3 Au moins depuis les romantiques allemands (notamment Johann Paul Richter), on sait que l'humour est un remède à la mélancolie. Mais dans bien des cas, cette fonction est inséparable du rythme qu'elle introduit dans la conversation et dans la vie sociale. Ainsi, pour Marcel Mauss, si l'usage de la plaisanterie en société s'explique par «le besoin de détente pour reposer d'une tenue par trop compassée ", il permet aussi d'établir " un rythme qui fait se succéder sans danger des états d'âme contraires " (Mauss, 1968-1969, 155). Pour sa part, Gaston Bachelard imagina la pratique d'une nouvelle discipline appliquée, la "rythmanalyse » qui, en s'appuyant notamment sur l'humour et le rythme, aurait visé « à rendre heureuses et légères les ambivalences que les psychanalystes découvrent dans les psychismes troublés» (Bachelard, 1957, 72). Cette idée me semble propre à nous faire redécouvrir certaines pratiques traditionnelles qui existaient bien avant l'époque contemporaine : par exemple, dans la civilisation arabo-musulmane médiévale, l'humour contribuait à rythmer les affects au cours des séances littéraires (Bouhdiba, 1979, 163-164).

Il s'agit donc ici de s'intéresser au rôle joué par la plaisanterie dans ces veillées nocturnes, samra. Dans un premier temps, je décrirai succinctement les veilléesde mariage. Mais pour examiner plus profondément les changements de rythme introduits par l'humour dans les interactions sociales et musicales, je présenterai le déroulement d'un type particulier de samra où j'ai pu observer au plus près le rôle de la plaisanterie comme issue, théâtralisée et rythmée, à la transe esthétique d'un musicien.

\section{Les veillées musicales et dansées, samra}

5 Habituellement, les veillées de mariage commencent après le cortège nuptial, ziffa, qui se déroule à l'extérieur de la maison du père du marié, après la prière du soir. Dans le passé, on se réunissait dans cette maison, mais depuis le milieu des années quatrevingt-dix, les fêtes de mariage ont lieu dans des tentes dressées dans la rue, ou dans des salles de fêtes aménagées à cet effet. Les invités s'installent vers 21 heures dans le salon ou sous la tente, sur des matelas, des coussins et des accoudoirs. Ils entourent le marié ${ }^{2}$ et sont accompagnés de plusieurs musiciens, poètes, bons danseurs et boute-en-train patentés. Pour les membres de la communauté villageoise ou de quartier, il s'agit de passer toute la nuit à se divertir. Dans le passé, ces festivités duraient trois nuits de suite, mais aujourd'hui, elles tendent à être réduites à une seule nuit. Comme le magyal, la samra est un rassemblement d'hommes semi-public, mais plus ouvert, avec une assistance beaucoup plus nombreuse. Elle n'est pas régie par un cérémonial aussi strict : l'atmosphère y est festive et joyeuse, la consommation du qat y est optionnelle, le comportement individuel est plus informel, la manière de s'asseoir est moins normative, et la priorité donnée à la danse contribue à libérer les corps de certaines contraintes sociales. Les formes cérémonielles entourant la musique sont à peu près les mêmes que dans le magyal, mais la plaisanterie alterne très souvent avec la musique.

Dans ces veillées nocturnes, les interventions musicales sont soit le fait des chantres religieux, nashshâd, qui interprètent des chants dévotionnels, nashîd, a cappella, soit le fait des chanteurs et instrumentistes profanes s'accompagnant du luth et jouant un répertoire plutôt profane, ghinâ. Après chacune de leurs suites chantées, certains chantres religieux avaient l'habitude, jusque dans les années quatre-vingt-dix, 
d'improviser eux-mêmes des intermèdes comiques afin de détendre l'atmosphère : parodies et imitations vocales de musique instrumentale, de la fanfare militaire de l'Imam, etc. Après les suites instrumentales, les plaisanteries sont plutôt improvisées par l'auditoire qui taquine gentiment le musicien.

D'autres types de plaisanterie sont pratiqués, qui n'incluent pas la musique : des défis verbaux, des imitations de présentateurs de télévision, des happenings joués par un faux aveugle ou un faux dévot. Dans beaucoup de ces plaisanteries, on remarque l'importance du procédé du pince-sans-rire: le ressort du comique est alors l'estompage de la limite "entre le sérieux et la plaisanterie », selon une expression arabe consacrée. Les plus efficaces de ces jeux sont ceux qui, en laissant planer cette ambiguïté, défient l'honneur d'une personne qui est alors prise à partie de plus en plus vivement ; la révélation, le plus tard possible, du " pour de rire » se produit aux dépens de cette personne, surtout si celle-ci a eu la faiblesse de prendre le défi au sérieux (souvent par une explosion de colère qui est risible en elle-même...) ${ }^{3}$. Ce modèle de transformation graduelle, masquant pour un temps un changement de sens qui se révèle ensuite soudainement, nous rappelle les transitions à la fois cérémonielles et musicales aménagées dans les parcours obligés exposées plus haut. Nous y reviendrons.

Dans ces séances musicales nocturnes, la question se pose plus particulièrement de savoir quelle faculté a l'humour d'impulser une nouvelle dynamique aux interactions succédant à la pratique musicale et à l'émotion esthétique. Il s'agira ici d'être attentif aux dimensions performatives et théâtralisées de l'expression verbale et gestuelle, notamment aux formes que prend la transition entre la musique et le retour aux interactions verbales ordinaires.

$\mathrm{Si}$, dans mes recherches précédentes, j'avais effectué de nombreux enregistrements musicaux, les plaisanteries n'avaient que peu attiré mon attention. Lorsque je commençai à m'y intéresser, leur compréhension linguistique s'avéra ardue, car elles recouraient à la langue parlée et dialectale, ainsi qu'à de nombreuses allusions symboliques ou personnelles. Or une des principales difficultés de l'ethnographie des mots d'esprit, c'est de ne guère pouvoir les faire répéter, car le mécanisme de l'humour perdrait alors toute sa spontanéité. De plus, les enregistrer était particulièrement difficile : touchant souvent à des normes dominantes de la société comme l'honneur et la religion, et à leurs points sensibles comme le corps et le sexe, les plaisanteries faisaient l'objet d'un tabou évident (souvent, on me demandait d'éteindre mon appareil enregistreur juste après la musique...). J'étais donc à la recherche d'un cadre plus favorable pour pouvoir les documenter. L'occasion m'en fut offerte par des séances de samra moins publiques et plus intimes.

\section{Un marathon nocturne de musique et d'humour}

10 À la fin des années quatre-vingt, puis à la fin des années quatre-vingt-dix, j'avais participé chez le chanteur et luthiste Yahyâ al-Nûnû à de longues samra organisées dans son petit salon. Comme les veillées de mariage, ces séances duraient toute la nuit, mais à la différence de celles-ci, elles ne répondaient pas à une occasion sociale ou religieuse particulière ; la musique y était l'activité principale (mais beaucoup moins la danse), et l'auditoire était assez restreint. Par ailleurs, il n'y avait pas d'interruption au coucher du soleil comme dans les fêtes de mariage, si bien que la séance durait du début de l'après-midi jusqu'au lendemain matin... Pour Yahyâ al-Nûnû, passer toute la 
nuit ensemble était une condition essentielle : il s'agissait de prolonger une tradition qu'il savait être en déclin, dans une société yéménite urbaine en pleine modernisation. Chez lui, la veillée était donc en train de se transformer en une épreuve d'endurance (où le qat nous aidait à tenir le coup) et en une pratique culturelle élitiste.

11 Pour moi, ces séances plus restreintes furent l'occasion d'étudier le rôle que l'humour joue dans ce type d'interactions sociales et artistiques, dans des conditions d'observation plus propices que dans les veillées de mariage communes: chez Yahyâ al-Nûnû, nous étions entre amis, une relation de confiance avait été nouée. À la fin des années quatre-vingt-dix, plusieurs prises de vue vidéo furent assurées par Pascal Privet, qui entretenait aussi de très bonnes relations avec lui. Le fait qu'il s'agisse d'un type de samra un peu particulier ne me paraissait pas un obstacle méthodologique rédhibitoire, même s'il me faudrait bien sûr en tenir compte dans l'analyse le moment venu.

Par ailleurs, il se trouve que, chez Yahyâ al-Nûnû, l'émotion créatrice, wujdân ${ }^{4}$, qui l'animait lors de ces marathons musicaux, s'exprimait d'une manière particulièrement intense que j'ai qualifiée de "transe émotionnelle» ou «transe esthétique»: s'il percevait chez ses auditeurs ce qu'il considérait comme une bonne qualité d'écoute, son chant et son jeu instrumental se bonifiaient, se diversifiaient, mais d'une manière qui, de son propre aveu, lui échappait. Il se mettait alors, selon ses termes, en " pilotage automatique ». L'émotion esthétique ressentie par ses auditeurs lui était renvoyée, selon une relation non verbale et involontaire, mais cumulative, qualifiée par lui « de cœur à cœur». Il était alors dans une phase d'inspiration qui, sur le plan des interactions ordinaires, l'éloignait de nous : cela se manifestait par diverses formes d'agitation du corps et d'expression orale extraverties, verbalisées ou non verbalisées. Mais sur le plan musical, cette relation ineffable le rendait d'autant plus présent: effectivement, il jouait et chantait de mieux en mieux au fil de la soirée, sans doute grâce à cette quasi-osmose avec son public. C'est la dissociation entre cet éloignement social et ce rapprochement musical que j'appelle ici «transe ». Or du fait qu'al-Nûnû avait une théorie assez élaborée sur l'effet de sa musique sur ses auditeurs, mais aussi à propos de sa propre émotion, il souhaitait tester ces phénomènes, un peu comme dans un laboratoire.

13 Ce fut pour moi l'occasion d'en faire une micro-ethnographie avec son plein consentement (Lambert, 2010). À cette occasion, je constatai l'interaction directe qui se produisait entre l'émotion musicale et les plaisanteries (comme dans les autres samra, mais d'une manière plus marquée). En effet, il semblait que l'atmosphère émotionnelle très chargée de ces séances ne pouvait se conclure harmonieusement que par la présence d'un humoriste qui, en faisant des plaisanteries après chaque chanson, la transformait totalement, dans un sens de convivialité et d'apaisement (dans le cas contraire, elle se concluait de manière déprimée et lugubre). Dès lors, je souhaitai prolonger mes réflexions précédentes sur les relations entre les changements de rythmes sociaux, et les changements de rythmes musicaux du magyal, par la prise en compte de l'humour dans un schéma plus général d'intelligibilité de la temporalité de ces séances cérémonielles, incluant à la fois leur partie diurne et leur partie nocturne, "jusqu'à l'aube », selon l'expression locale consacrée. J'y avais été encouragé par une appellation que leur donnaient certains mélomanes: des "séances abbassides", expression sans doute inspirée par le Livre des chansons d'Isfahânî, qui racontait les 
séances musicales nocturnes se déroulant à Bagdad à l'époque abbasside, l'âge d'or de l'islam (Sawa, 1989, 111).

14 Je suivis donc certaines de ces séances nocturnes, en général chez Yahyâ al-Nûnû, qui était accompagné du percussionniste Ahmed 'Ushaysh, vers 1995, puis, à partir de 1997, du percussionniste Mohammed al-Khamîsî. Musicien et poète hauts en couleur, al-Khamîsî avait l'habitude d'accompagner al-Nûnû avec son plateau en cuivre, le sahn, de reprendre en chœur certains refrains, de battre des mains et de l'encourager par des interventions verbales diverses. En général, l'auditoire était constitué d'al-Khamîsî, un ou deux auditeurs yéménites, un ou deux auditeurs étrangers, le cinéaste et moi-même. Manifestement, cette situation ne laissait pas indifférent Yahyâ al-Nûnû qui, tout en étant en transe, pouvait aussi fixer la caméra d'une manière très cabotine...

\section{Des bénédictions qui dégénèrent en plaisanteries}

Il me faut rappeler que, dans la samra comme dans le magyal, les suites musicales successives s'insèrent selon une rythmique cérémonielle graduelle. Ainsi, à la fin de la prestation musicale, on n'applaudit pas ${ }^{5}$, mais on psalmodie une prière collective de bénédiction, sous une forme conventionnelle et à laquelle participe le musicien. Cette prière implore la protection divine pour le prophète Mohammed qui y est désigné par un pronom personnel (et un adjectif possessif) à la troisième personne :

Allah, je Te prie pour lui et pour son salut

Puisse Dieu être prié pour lui et pour son salut et pour celui de sa famille. Amen ${ }^{6}$. toujours mentionné par son nom dans les deux derniers vers du dernier poème chanté7. Il y a donc une continuité sémantique entre la poésie chantée et cette prière. Sur le plan sonore, la prière peut être considérée ici comme une forme intermédiaire entre la musique et la voix parlée, au moyen de cet hybride que représente la psalmodie : une parole religieuse syllabique, à peine mélodisée, dont la corde de récitation repose sur la même note que la tonique de la dernière chanson. À la suite de la prière collective, chacun félicite individuellement le musicien avec la formule suivante : « Il a été doux et il a préservé ! " Le musicien répond de manière aussi conventionnelle: "Que Dieu adoucisse votre vie ! », ou bien : « Vous êtes la douceur même ! ${ }^{9}$ »

Ces formules de bénédiction qui suivent la prière signalent donc un retour graduel à la parole ordinaire, après que le musicien et les auditeurs ont été emportés dans un monde sonore où cette dernière n'a pas cours. On peut résumer cette succession de la façon suivante :

Figure $n^{\circ} 1$

\section{Suite musicale qawma}

\section{Prière collective de bénédiction}

\section{Compliments en forme de bénédiction}

Dans les samra organisées par Yahyâ al-Nûnû, c'est à ce moment que Mohammed al-Khamîsî intervenait plus particulièrement. Dans ces instants inoubliables où al-Nûnû venait de cesser de chanter et de jouer, il était habité par une tension émotionnelle extrême. Après la prière et les formules de bénédiction, 
al-Khamîsî enchaînait des félicitations qui prenaient graduellement la forme de plaisanteries. Je les décrirai ci-après telles qu'elles ont été enregistrées sur quelques documents vidéo ${ }^{10}$.

19 À la fin de la partie chantée, Yahyâal-Nûnû conserve son luth, et Mohammed al-Khamîsî psalmodie la prière de bénédiction. Al-Nûnû étreint alors son instrument, face contre lui; il l'embrasse et lui dit des mots tendres, la voix chevrotante d'émotion: «Mon chéri!» (Yâhabîbî), «Mon enfant!» (Yâwaladî). Il semble éploré et ravi à la fois. En considérant ainsi son instrument comme son fils, Yahyâ al-Nûnû reprenait un mythe d'origine attribuant l'invention du luth à Lamek, un personnage biblique qui en avait fabriqué un avec les membres de la dépouille de son fils mort (al-Mufaddal Ibn Salâma, cité par Robson, 1938, 239).

Pendant ce temps, al-Khamîsî commence à faire des compliments à al-Nûnû sous forme de bénédictions personnalisées : "Que ta main soit préservée !» [sous-entendu : qu'elle aille au paradis, c'est-à-dire après la mort]. Ceci est une allusion au statut méprisé des musiciens dans la société yéménite car, dans le passé, ils étaient menacés de l'enfer par les religieux.

21 Al-Khamîsî reprend quelques paroles de la chanson, cette fois en les déclamant : « En te disant adieu, je te confie à Allah». Ensuite, ses commentaires deviennent progressivement plus taquins : prenant l'auditoire à témoin, en parlant du musicien à la troisième personne: "Il est troublé, il a la tête sens dessus dessous (marbûsh). Que d'amour ! Il n'y a de Dieu que Dieu !». Puis à un auditeur : « Ne t'en fais pas, continue à mâcher ton qat! » Graduellement, al-Khamîsî crée une certaine mise à distance de la situation : « Le soleil va se lever dans pas longtemps, et nous, nous sommes encore là à être assis! » Ici, il se moque clairement de la longueur des séances nocturnes qui lui est imposée par Yahyâ al-Nûnû...

22 Une autre séquence ${ }^{11}$ illustre la progression des plaisanteries. Après une intervention musicale, Yahyâ al-Nûnû ayant posé son luth, Mohammed al-Khamisî psalmodie la prière de bénédiction et lance quelques piques: «Bravo pour tes péchés! $»^{12}$ [à qui s'adresse cet étrange compliment : au musicien ? à l'un des auditeurs ? Son ambigüité est sans doute liée au statut du musicien dans la société, plutôt méprisé...]; «Au secours, ô mon Dieu!»

Puis il le taquine explicitement:

Bravo, Père la Calotte! [Yahyâ porte une calotte sur la tête, signe extérieur de religiosité] Que le manteau protecteur d'Allah nous recouvre, ô Prophète de Dieu! Que tu sois absous (pour tes péchés), et qu'ils le soient tous eux aussi! » [tout en incluant d'un grand geste les auditeurs présents; cette fois-ci, les destinataires sont explicitement désignés].

23 Al-Khamîsî continue à passer insensiblement de formules cérémonielles, qu'il décline à sa façon, à des compliments teintés d'ironie, prenant l'auditoire à témoin : "C'est incroyable! Il (al-Nûnû) va rester comme ça jusqu'à ce qu'il le pose (le luth). On n'a jamais vu ça et on ne reverra jamais ça!». Et il conclut en s'adressant à un auditeur : « Reçois de moi et de lui soixante-dix-sept baisers! » [avec une mimique cocasse et les trois premiers doigts joints puis projetés en avant, imitant le mouvement des lèvres avec sensualité, tout en s'esclaffant...].

Souvent, le sujet de ces plaisanteries glissait progressivement en-dessous de la ceinture. Après une autre suite musicale al-Khamîsî s'adresse à al-Nûnû en égrenant les commentaires suivants : 
- Regarde ce que tu nous as fait! Tu as dépeuplé (khala't) notre monde !- Je vais dormir cul par-dessus tête!

- Ah, je me demande par où les djinns viennent te chatouiller?! ! [gestes comiques des doigts, orientés vers la partie inférieure du corps d'al-Nûnû...].

- Et c'est comme ça que tu nous savonnes la verge ?! !13

Al-Khamîsî fait se succéder ces phrases exclamatives dans une sorte de monologue, d'une manière plus ou moins espacée, selon l'inspiration, tantôt avec emphase, soulignant la sonorité des mots, tantôt d'une voix caverneuse typique des artisans et des hommes de tribus lorsqu'ils abordent des sujets graves, et tantôt d'une voix neutre comme s'il parlait à un animal familier dont il n'attendrait pas de réponse (al-Nûnû ne répond d'ailleurs quasiment pas). Encouragés par les blagues d'al-Khamîsî, les autres auditeurs lancent aussi des compliments et quelques plaisanteries au musicien.

\section{Apostrophes parodiques par antiphrase}

Il existe aussi dans la culture de Sanaa une forme de plaisanterie consistant à s'adresser à l'autre sur un mode sarcastique ou par antiphrase, en transformant par exemple une formule de bénédiction en malédiction ${ }^{14}$. Les compliments adressés aux musiciens ont raffiné ce type d'expression sous la forme d'une apostrophe. À ce propos, un autre accompagnateur de Yahyâ al-Nûnû, Ahmed 'Ushaysh, me racontait, concernant un grand musicien disparu dans les années soixante :

Après qu'Ahmed Fâyie' avait fini de chanter, nous délaissions les formules habituelles et nous lui lancions des blagues. Comme il était surnommé « la souris » parce qu'il était tout menu, on lui disait :

$-\mathrm{O}$ toi qui fais des trous dans les toits!

- $\mathrm{O}$ toi qui chies au-dessus de la mélasse! [allusion à une souris qui, n'osant pas descendre dans un pot de mélasse pour la manger, resterait perchée sur le bord du pot, tremperait sa queue dedans, puis la lécherait...]

Cette forme d'adresse à la deuxième personne ( Ô toi qui... ») est une sorte de pastiche de la poésie courtoise où ce type d'interpellation solennelle est un trope très courant pour s'adresser à la bien-aimée : «Ô toi qui as retiré le sommeil de mes yeux... ». C'est également le cas dans la poésie religieuse de louange à Dieu : « Ô Toi sur Qui nous nous appuyons... ». Mais ici, le décalage parodique met le destinataire à une place qui n'est pas la sienne, tout en lui attribuant un comportement trivial et contraire aux normes littéraires ou religieuses. Cette inversion des valeurs, le musicien s'en trouve en quelque sorte tenu pour responsable. C'est sans doute aussi sa marginalité par rapport aux normes dominantes qui autorise cette licence ${ }^{15} \ldots$ Mais dans ces apostrophes, l'apparente ironie, parce qu'elle est tempérée par le raffinement poétique et l'imagination, permet d'exprimer l'affection et l'admiration pour le musicien sous le masque du sarcasme, donc avec une certaine pudeur (dans une culture où l'on n'exprime pas facilement ses véritables sentiments, surtout entre hommes). Leur aspect paradoxal est, au fond, plein de bienveillance.

Ce témoignage nous confirme que le traitement du compliment par l'humour était assez répandu dans la culture ancienne des samra. Pour al-Khamîsî comme pour 'Ushaysh, ces communications indirectes permettaient d'exprimer leur respect pour l'art du musicien beaucoup plus sûrement que ne l'aurait fait un banal discours positif du type : « Bravo, j'aime ta musique ». Ici, l'important était de ne pas chercher à tirer le musicien de son état émotionnel sur un mode discursif et rationnel. De telles phrases 
maladroites que j'avais entendu émettre par des auditeurs yéménites (ou que j'avais parfois émises moi-même) ne faisaient qu'exaspérer le sentiment de solitude et de frustration de Yahyâ al-Nûnû, car il les prenait pour un manque de reconnaissance envers son implication personnelle et sa sincérité artistique. Dans les occasions où il n'y avait eu personne pour provoquer al-Nûnû par des plaisanteries, j'avais pu constater que la séance se terminait presque toujours dans une tonalité dramatique et maussade. En revanche l'antiphrase et l'ironie étaient acceptées, voire encouragées, sans doute parce qu'en tant que communication symbolique, elles répondaient le mieux à cette autre communication symbolique qu'est la transe musicale.

\section{Des saynètes grivoises improvisées} al-Nûnû le retient et commence à broder sur le thème de l'épouse d'al-Khamîsî « qui doit être en train de l'attendre à la maison avec impatience » : comme il va rentrer tard, " elle va lui passer un savon ». Cherchant à retarder al-Khamîsî, al-Nûnû insiste pour que celui-ci mange encore des gâteaux secs et boive du thé, de façon que, n'ayant plus faim, « il ne pourra pas manger le dîner que sa femme va lui présenter » (ce qui, dans les relations de couple au Yémen, peut être un casusbelli...). Il asperge abondamment al-Khamîsî de parfum, et imagine que sa femme va lui poser toute une série de questions, dont celle - inévitable - du lieu où il a passé tout son temps. Et il pousse la plaisanterie jusqu'à imaginer al-Khamîsî dépourvu de désir (à cause du qat et de son grand âge), ne pouvant répondre aux ardeurs de sa femme (beaucoup plus jeune que lui) et ne pouvant que lui dire : «Contente-toi de me renifler ! (il fait alors allusion à l'encens et au parfum généreusement dispensés).

31 Pour sa part, al-Khamîsî se prête au jeu, abondant dans le sens d'al-Nûnû, par autodérision, jouant les scènes de manière burlesque, avec des gestes cocasses, des bruitages grotesques. Le style d'énonciation de ces échanges improvisés est très particulier (par rapport à une conversation ordinaire) : étant encore imprégné de la transe, al-Nûnû débite les épisodes de farce d'une manière très inspirée, selon un rythme presque haletant, dans une sorte de logorrhée irrépressible, en s'esclaffant au fur et à mesure de ses propres trouvailles, dans un état de jouissance très enfantin, presque puéril... En fait, la transe se poursuivant, elle a pris une coloration différente. Il faut dire que l'épuisement physique en fin de nuit contribue à ce relâchement relatif...

La relation entre les deux compères est très complémentaire. La dimension sexuelle n'est pas exempte de significations : en se moquant d'al-Khamîsî, et en imaginant que c'est sa femme qui va prendre l'initiative, al-Nûnû inverse les rôles sexuels. Il n'est évidemment pas anodin que al-Khamîsî soit, du moins dans ce contexte, un simple percussionniste $^{16}$, destiné, depuis le début de la séance, à servir de faire-valoir au soliste, et prêt à subir toutes ses avanies... Peut-être qu'en lui permettant d'inverser les 
rôles dans la plaisanterie, al-Khamîsî offre au chanteur instrumentiste la possibilité de renverser les stéréotypes dont ce dernier est l'objet dans les représentations collectives dominantes, et de se repositionner ainsi en imagination dans une position plus avantageuse.

\section{Sortir graduellement de la transe par le rire}

Dans l'ensemble des interactions présentées, les plaisanteries adressées au musicien par son commensal sont clairement un moyen de le faire sortir de cette expérience émotionnelle à la fois intime et extrême qu'est la transe esthétique, d'une manière graduelle, conviviale et socialement acceptée. Al-Khamîsî prenant d'abord l'initiative de la plaisanterie, fait monter celle-ci en puissance tout en introduisant à la fois de la distanciation et de la provocation, jusqu'à ce que al-Nûnû, enfin déridé, prenne à son tour l'initiative. À des moments plus calmes, al-Nûnû m'avait d'ailleurs confirmé qu'il faisait volontairement appel à Ahmed 'Ushaysh et à Mohammed al-Khamîsî pour le seconder dans ces longues veillées musicales.

À cette fin, l'émotion du musicien est «traitée » par un humour d'abord unilatéral à la suite de chaque performance musicale, puis devenant progressivement « interactif ", à la fin de la séance nocturne. Cette forme d'expression indirecte commence par faire sourire al-Nûnû, et dissipe la tension émotionnelle dans laquelle il s'est plongé. C'est aussi un moyen pour les auditeurs de lui exprimer leur émerveillement de manière sensible et raffinée. Chez al-Khamîsî, cela se joue à la fois dans la théâtralisation, dans l'alternance de différents types de voix (emphatique, caverneuse, blanche) et dans le déploiement temporel marqué par des ruptures linguistiques et performatives savamment tuilées.

Pour s'adresser au musicien et aux participants, Khamîsî passe alternativement de la deuxième à la troisième personne, voire à la première personne du pluriel. Un peu comme un récitant, il instaure une distanciation "scénique " et une réflexivité en distribuant implicitement des rôles aux participants : acteurs, spectateurs, ou tantôt l'un et tantôt l'autre. Il tisse aussi des liens entre eux: "Reçois de moi et de lui soixante-dix-sept baisers!» L'interaction sociale devient spectacle éphémère. On notera d'ailleurs que la plaisanterie, notamment la saynète de la fin, partage avec la transe une dimension théâtrale et une dimension narrative (dans la transe, le mythe d'origine du luth, rejoué implicitement par Yahyâ al-Nûnû) ${ }^{17}$. En revanche, si la transe visait à rapprocher de manière fusionnelle les auditeurs du musicien, à ne faire d'eux, idéalement, qu' " un seul cœur », pour sa part, la plaisanterie crée une distance qui les sépare grâce à l'entremise de ce tiers qu'est le plaisantin. Il s'agit d'une forme de médiation par excellence ${ }^{18}$.

Comme on l'a vu, il y a, en général dans les samra organisées par al-Nûnû, un crescendodans le comique, dans la provocation et dans la levée des tabous. Dans la séquence 1, Khamîsî se moque gentiment du musicien. Dans la séquence 2, il commence à le défier en lui inventant des sobriquets. Dans la séquence 3, le comique devient salace. Dans les saynètes de fin de nuit (moins fréquentes), la grivoiserie est clairement partagée. Certes, les séquences présentées ici sont tirées de soirées différentes, mais elles restituent assez fidèlement ce processus que j'ai pu observer régulièrement lors de nombreuses veillées. Or c'est bien ce relâchement (relatif) qui déclenche le 
retournement d'al-Nûnû et, à un moment donné, lui fait prendre l'initiative de la plaisanterie : il y trouve une véritable issue à la tension de son état psychique.

Ce crescendo est servi par des transitions sémantiques et performatives imperceptibles: les premières plaisanteries sont des variations sur les formules religieuses et rituelles, glissant du pastiche à la parodie. Les prières acquièrent un double sens comique, tandis que les plaisanteries prennent souvent la forme d'invocations (à Dieu, au Prophète), introduisant une délicieuse ambigüité. La narration comique (sous forme de farce, mais aussi sous forme d'histoires drôles) représente une étape supplémentaire. Cette progression peut être résumée par le schéma suivant :

Figure $\mathrm{n}^{\circ} 2$

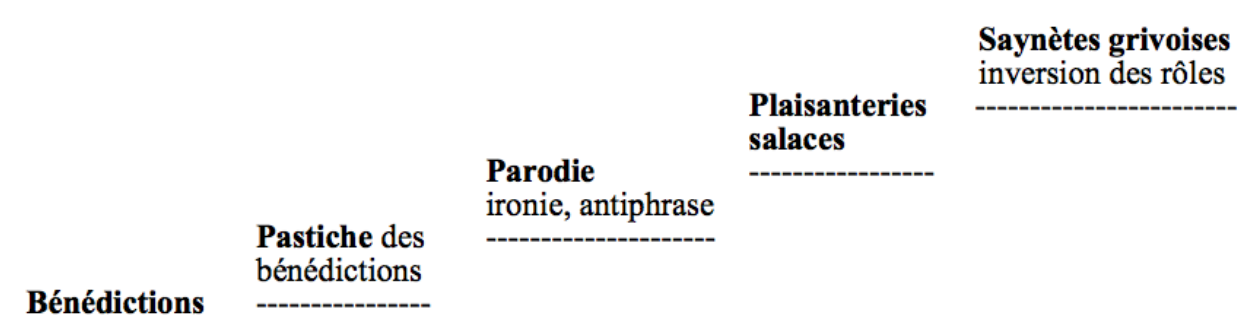

La succession des plaisanteries et leur crescendo C'est un amuseur, mais pas du tout anecdotique : il est non seulement un médiateur dans la performance, mais aussi un commutateur, transformant progressivement la relation entre l'artiste et ses auditeurs, mais aussi celle de l'artiste avec lui-même. Il le fait en utilisant les ressorts temporels et rythmiques d'une certaine théâtralité : énonciation rhétorique des plaisanteries selon des débits différents, égrainées, déclamées, exclamées, sous forme de litanie (et al-Nûnû sous forme de logorrhée), et surtout aménageant des transitions dont certaines sont progressives (montée en puissance de la grivoiserie) et certaines soudaines (inversions des rôles dans les apostrophes paradoxales et dans la saynète improvisée).

Comme la transe, la plaisanterie est une forme d'expression à la fois émotionnelle et symbolique. Dans les deux cas, il s'agit d'une communication indirecte et performative : l'humour agonistique des interpellations poétiques, de même que l'excès émotionnel de la transe (Lambert, 2010, 160), a pour fonction d'attester de la sincérité de l'expression des sentiments. Mais alors que la transe tendait à établir une fusion ineffable des affects entre les participants, la plaisanterie, elle, dissocie à nouveau les rôles et rétablit entre les individus une distance socialement et verbalement acceptable, que l'on pourrait qualifier de simple complicité. Le jeu de rôles est donc aussi un facteur de transmutation, il contribue à atténuer la transe en évitant d'aborder frontalement ses manifestations excessives. On comprend ainsi comment al-Khamîsî peut se prêter si facilement au rôle de faire-valoir : c'est un rôle psychologique et social indispensable à la bonne conclusion des séances. 


\section{Conclusion : plaisanterie et rythmanalyse} l'enchaînement des bénédictions puis des différentes formes de plaisanterie nous montrent le déploiement d'une technique achevée de la transition et des changements de rythme et de sens dans les interactions cérémonielles et comiques (figure 2). On retrouve ici la continuité, la progression et l'irréversibilité qui, au Yémen, sont caractéristiques des " parcours obligés » musicaux et sociaux. Les transitions tendent à masquer le plus longtemps possible l'inversion du sens (sur le mode du pastiche et du pince-sans-rire) - jusqu'à ce que cette dernière se dévoile soudainement (dans la parodie, les compliments ambigus et les apostrophes par antiphrase), déclenchant le ressort même du comique. Simultanément, comme le montre al-Khamîsî dans l'une de ses saillies («Le soleil va se lever, et nous, nous sommes encore là... »), la plaisanterie réintroduit un temps social linéaire, inverse de la conception atomiste du temps propre à la musique et à la jouissance esthétique de l'instant (Lambert, 2004, 164).

nous reprenons cette réflexion à son point initial, nous pouvons noter que la dynamique des transitions imperceptibles (les «tuilages») puis de l'inversion ou transformation soudaine du sens, qui est présente dans les interactions cérémonielles du magyal d'après-midi, trouve une nouvelle réalisation dans la samra nocturne, bien que d'une manière un peu différente: la plaisanterie y occupe une place plus importante, car au lieu de ne faire qu'introduire à la séance, comme dans le magyal, elle est convoquée presque après chaque intervention musicale, que ce soit du chant religieux ou de la musique profane. Il s'agit donc d'une sorte de relance, de nature cyclique, où cette séquence particulière - musique + transe + bénédictions + plaisanteries - (approximativement, figures $1+2$ ) est comme un épisode récurrent des veillées nocturnes, samra. De plus, à chaque occurrence de cette séquence, il se produit un crescendo à la fois dans l'émotion musicale et dans l'excitation comique, jusqu'à la saynète finale. La plaisanterie contribue donc à ponctuer les veillées, à les développer dans le temps, bref, à les rythmer, tantôt de manière cyclique et tantôt de manière linéaire.

À présent, si nous envisageons la samra non comme une veillée séparée, mais comme la prolongation d'un magyal diurne, les deux formant ensemble un seul long cérémoniel, cet apogée nocturne du comique vient en quelque sorte boucler le cycle très long qui, comme on l'a vu, commence, en début d'après-midi, là aussi par des plaisanteries. Au moins dans le cas particulier de ces "veillées-marathon", l'enchaînement des plaisanteries est comme le couronnement d'un processus plus général.De sorte que l'on pourrait résumer le déroulement de ces séances «jusqu'au matin » en disant qu'elles commencent et qu'elles finissent par des plaisanteries, après être passées par toute une gamme d'émotions, de sentiments et de pratiques expressives parfois contradictoires (avec glissements et inversions des rôles). Certes, on sent bien que ce modèle des fameuses "séances abbassides" s'applique plus facilement aux veillées élitistes de Yahyâ al-Nûnû qu'aux samra de mariage les plus communes, mais il semble cependant qu'il y a là des structures profondes qui sont à l'œuvre, dans des proportions différentes selon chaque type d'événement. Pour reprendre les termes de Marcel Mauss, ces séances se déroulent selon « un rythme qui fait se succéder sans danger des états d'âme contraires ». 
entendu, on ne saurait aborder cette pratique de l'humour et de la musique uniquement à travers sa fonction sociale: à la différence des autres happenings comiques des soirées de mariage, il s'agit ici d'un humour réservé à des circonstances intimes et à une élite culturelle, où la dimension individuelle est importante. Dans quelle proportion l'ambigüité entre le comique et le sérieux exprime-t-elle des enjeux sociaux ou une part de génie personnel ? Il est difficile de le dire avec précision. Mais le fait que Yahyâal-Nûnû a toujours recherché la compagnie d'un percussionniste, successivement Ahmed 'Ushaysh puis Mohammed al-Khamîsî, pour jouer un tel rôle d'amuseur nous met peut-être sur une piste: il s'agit d'un art en soi, requérant des spécialistes. Or une telle maîtrise des interactions cérémonielles aurait-elle pu trouver de meilleurs spécialistes que ceux qui maîtrisent aussi le rythme musical ? Dès lors, ne doit-on pas penser que la société yéménite nous présente ici une version achevée de rythmanalyse où, selon les termes de Bachelard, la musique et la plaisanterie se combinent et se succèdent rythmiquement pour "rendre heureuses et légères les ambivalences » inhérentes à toute société?

\section{BIBLIOGRAPHIE}

BACHELARD, Gaston, 1957, Poétique de l'espace, Paris, PUF.

BATESON, Gregory, 1977, Pour une écologie de l'esprit 1, Paris, Seuil.

BECKER, Judith, 2005, Musique et transe, in Jean-Jacques Nattiez (dir.), Musiques :

uneencyclopédiepourleXXI ${ }^{e}$ siècle.3.Musiqueetcultures, Arles, Actes Sud, Cité de la Musique, p. 459-487.

BouHDIBA, Abdelwahhab, 1979, la Sexualité en Islam, Paris, PUF.

DURING, Jean, 1988, Musique et extase : l'audition mystique dans la tradition soufie, Paris, Albin Michel.

HENNION, Antoine, 2007, la Passion musicale : une sociologie de la médiation, Paris, Métailié.

LAMBERT, Jean, 1997, la Médecine de l'âme : le chant de Sanaa dans la société yéménite, Nanterre, Société d'ethnologie [photos, glossaire, index, 1 CD encarté].

LAMBERT, Jean, 2004, Temps musical et temps social au Yémen : la suite musicale dans le magyal de Sanaa,l'Homme, « Musique et anthropologie », 171-172, p. 151-172.

LAMBERT, Jean, 2010, le Musicien Yahyâ al-Nûnû. L'émotion musicale et ses transformations, Cahiersd'Ethnomusicologie, 23, p. 147-171.

LORTAT-JACOB, Bernard, 1987, l'Improvisation : le modèle et ses réalisations, in B. LORTAT-JACOB (éd.), l'Improvisation dans les musiques de tradition orale, Paris, SELAF, p. 45-59.

MAUSS, Marcel, 1968-1969, Parentés à plaisanterie Euvres. 3. Cohésion sociale et division de la sociologie, Paris, Les Éditions de Minuit, p. 109-124.

ROBSON, James, 1938, The Kitâb al-malâhî of Abû Tâlib Al-mufaddal ibn Salâma, Journal of Royal Asian Studies, pp. 231-249.

Cahiers de littérature orale, 73-74 | 2013 
SAWA, George D., 1989, Music Performance Practice in the Early 'Abbasid Era, 132-320 ah/ 750-932 ad, Toronto, Pontifical Institute for Mediaeval Studies.

\section{NOTES}

1. Ce concept avait été inspiré à des ethnomusicologues par certains jeux de société comme le jeu de l'oie (Lortat-Jacob, 1987, 54-55).

2. Qui ne se retirera qu'en milieu de soirée pour rejoindre sa nouvelle épouse.

3. La théorie de ces jeux bâtis sur la question: «Est-ce un jeu ?» a été faite par Gregory Bateson qui les rapproche des brimades rituelles d'initiation (Bateson, 1977, 214).

4. Ne pouvant développer ce concept de wujdân dans le cadre restreint de cet article, je renvoie le lecteur à la description qu'en fait Jean During, sous le synonyme de wajd dans le contexte du soufisme (During, 1988, 91 et sq).

5. C'était le cas jusque dans les années quatre-vingt-dix.

6. Allahummâ 'sallî wa-sallam 'alayh/Sallâ Allah wa-'sallam 'alayh wa-'alâ âlehe.

7. Par exemple: «Que soit pure (ta) prière/Sur leProphète d'Allah/Prie Allah pour lui/Tout au long des Temps».

8. Annas wa-haras!

9. Annas Allah bi-hayâtkum ; - Wa-antum al-uns !

10. Séquence vidéo $1: 1,08 / 97$, plage $9: 01: 48$ '. Prises de vue : Pascal Privet. Vidéo accessible à partir du site $:$ http://clo.revues.org.

11. Séquence $2: 1,08 / 97$, plage $1: 00: 11$ ' environ, $c f$. http://clo.revues.org.

12. Littéralement : « (Que Dieu te donne) la santé autant que (tu as commis) de péchés : Lek a-'âfiya fi dhunûbek!»

13. En 1995, cette séquence n'avait pas été enregistrée, ce qui peut en expliquer la plus grande liberté de parole.

14. Pour me faire un reproche euphémisé, un ami m'avait lancé : «Que Dieu déchire ton pyjama!»

15. Une telle inversion des valeurs est d'ailleurs bien reconnue comme le résultat de l'action de la musique, comme le montrent certaines formules d'al-Khamînî: : Je vais dormir cul par-dessus tête! ».

16. C'est seulement plusieurs années après que je m'aperçus que Khamîsî était aussi un grand chanteur soliste. Cette découverte tardive me montra, a posteriori, combien il jouait bien son rôle modeste !

17. Dans le soufisme, du moins dans le monde malais, l'entrée en transe d'un adepte correspond en général « au déclenchement d'un mode narratif particulier » (Becker, 2005, 475).

18. Sur le concept de médiation en sociologie de l'art et de la musique, voir Hennion $(2007,29)$. 


\section{RÉSUMÉS}

Dans la ville de Sanaa, au cours des veillées nocturnes de mariage, samra, la musique et la danse sont souvent suivies de plaisanteries qui favorisent l'engagement de tous les participants dans la réjouissance collective. Dans les samra plus restreintes qui se déroulaient chez le musicien Yahyâ al-Nûnû dans les années quatre-vingt-dix, la plaisanterie avait une fonction supplémentaire, celle de désamorcer son investissement émotionnel intense dans la musique. Dans les deux cas, les plaisanteries lancées par un comparse, en passant graduellement du sérieux à la grivoiserie, faisaient alterner des états mentaux les plus variés, contribuant à produire ce que Gaston Bachelard a appelé une rythmanalyse.

In the city of Sanaa, during the wedding night-gatherings, samra, the music and the dance are often followed by jokes which facilitate the commitment of all in the collective rejoicing. In the more restricted samra which were organized by the singer Yahyâ al-Nûnû in the nineties, the joke had an additional function, that to defuse his intense emotional investment in musical practice. The jokes were launched by a partner who was passing gradually from religious formulas to saucy expressions, throwing into the participants in the most varied mental states, and contributing to produce what Gaston Bachelard called a "rythmanalyse".

INDEX

Thèmes : ethnomusicologie

Index géographique : Yémen

Keywords : Yemen, Musical Emotion, Lute, Joke, Ceremonial, Theatricality, Rhythm

Mots-clés : émotion musical, luth, plaisanterie, cérémoniel, théâtralité, rythme

\section{AUTEUR}

JEAN LAMBERT

MNHM/CREM-LESC, UMR 7186, CNRS 